\title{
Diversidade de peixes em poças de um rio intermitente do semiárido paraibano, Brasil
}

\author{
Maria Marcolina Lima Cardoso * \\ Jane Enisa Ribeiro Torelli de Souza \\ Maria Cristina Crispim \\ Rosângela Siqueira \\ Centro de Ciências Exatas e da Natureza, Universidade Federal da Paraíba \\ Cidade Universitária, CEP 58050-900, João Pessoa - PB, Brasil \\ * Autor para correspondência \\ marcolinaipj@yahoo.com
}

Submetido em 04/10/2011

Aceito para publicação em 05/04/2012

\section{Resumo}

As regiões semiáridas do Nordeste brasileiro sofrem com os prolongados períodos de estiagem, afetando a qualidade da água e a produtividade pesqueira. Os estudos têm demonstrado que as alterações entre períodos de chuva e estiagem têm profundas influências sobre os organismos dos ambientes aquáticos das regiões semiáridas e em especial sobre a ictiofauna. Apesar disto, poucos são os estudos que tentam conhecer e elucidar a ecologia de peixes do semiárido. O objetivo do presente artigo foi conhecer a diversidade e alguns aspectos da estrutura populacional de espécies de peixes, a fim de avaliar a importância da formação das poças para a conservação das espécies e da produtividade pesqueira. Foi encontrado um total de 13 espécies de peixes, das quais uma espécie é exótica (Oreochromis niloticus). A abundância de espécies foi altamente variável entre os meses de coleta, embora a riqueza tenha revelado baixa flutuação. As espécies de importância econômica apresentaram tamanho médio acima de $7 \mathrm{~cm}$ de comprimento padrão e 57,9\% dos indivíduos estiveram em atividade reprodutiva. Os resultados demonstraram que o período de estiagem afeta a comunidade de peixes, sendo que as poças parecem ser locais importantes para a conservação da diversidade e da produtividade pesqueira.

Palavras-chave: Caatinga; Ictiofauna; Poças; Riqueza

\section{Abstract}

Fish diversity in pools of an intermittent river in a semiarid region of Paraíba, Brazil. Prolonged periods of drought cause alterations in water quality and productivity of fisheries in semiarid regions of Brazil. Changes between periods of rain and drought affect aquatic organisms, especially fish. However, there are few studies that have attempted to understand and elucidate the ecology of fish in these semiarid regions. The purpose of this study was to investigate the diversity, and some aspects of population structure of fish species in these areas, in order to evaluate the importance of shallow pond formations in the conservation of these taxa and the productivity of these fisheries. We recorded 13 fish species, of which one was exotic (Oreochromis niloticus). Species abundance was highly variable among months, while variability of richness was low. The average size of the fish, which is important for the local economy, was over $7 \mathrm{~cm}$ long, and $57.9 \%$ of individuals were 
reproductively active at the moment of capture. The results show that the dry season affects the structure of the fish community and that the pools are important sites for the conservation of the diversity and productivity of these fisheries.

Key words: Caatinga; Ichthyofauna; Ponds; Richness

\section{Introdução}

As regiões semiáridas do nordeste do Brasil sofrem consideravelmente com a escassez de água, passando por períodos longos de estiagem (oito a nove meses) e curtos períodos de cheia (três a quatro meses). Devido ao prolongado período de seca e ao solo raso, são poucos ecossistemas aquáticos que permanecem alagados durante todo o ano, criando-se o problema da falta de água, da baixa qualidade da água disponível para o consumo e a redução da produção pesqueira. Como forma de minimizar estes problemas, grande parte dos rios de maior porte são barrados para a formação de reservatórios, constituindo-se numa rica fonte de água e alimento para as populações dessas regiões (LACERDA, 2003).

No semiárido nordestino, a maior parte dos rios possui caráter intermitente e constitui uma importante fonte de água e alimento para as populações ribeirinhas (BARBOSA, 1998). Os organismos que habitam tais ambientes estão sujeitos a fortes mudanças na composição da água, uma vez que os rios intermitentes surgem rapidamente durante o período de chuva e logo desaparecem, restando apenas poucas poças temporárias, muitas das quais, em períodos de alta precipitação pluviométrica, podem permanecer alagadas.

As variações no fluxo de água decorrentes da seca e da cheia podem ser consideradas os principais fatores que influenciam os padrões e modelos de sucessão ecológica das comunidades aquáticas dos rios do semiárido (MALTCHIK, 1999; ABÍLIO et al., 2007). Segundo Medeiros (1999), a variação no fluxo de água pode afetar a comunidade de peixes por meio das alterações no habitat.

No início do período chuvoso, o rápido fluxo de água provoca o revolvimento do fundo do corpo aquático, promovendo o carreamento da matéria orgânica dos ambientes terrestres, que por sua vez, aumenta a turbidez da água (ABÍLIO, 2002). Com a diminuição da intensidade de chuvas, a água desses ambientes torna-se menos turva, e, durante o período de seca, com o baixo volume da água, muitas vezes, ocorre a eutrofização natural, devido à alta concentração de nutrientes como fósforo e nitrogênio (VIEIRA et al., 2009).

Assim como outros corpos da água, as poças que são formadas durante o período de seca estão sujeitas a eutrofização, e, apesar das condições adversas que ocorrem durante este período, incluindo a baixa disponibilidade de alimentos, redução do oxigênio dissolvido e pouco espaço para locomoção (MATTHEWS, 1998), algumas espécies de peixes ainda persistem nas poças. Portanto, é provável que estes ambientes aquáticos possam servir como importantes locais de desova para várias espécies, promovendo um papel fundamental na recolonização dos seus estoques (MEDEIROS; MALTCHIK, 2000).

Além das condições severas impostas pelo ciclo hidrológico, grande parte dos ambientes aquáticos do semiárido tem sido alterada pela introdução de espécies exóticas, considerada como a segunda maior causa da diminuição da diversidade de espécies (VITOUSEK et al., 1997), como também, pela eutrofização artificial e assoreamento. Apesar das características singulares dos ambientes semiáridos, há poucos estudos sobre os ambientes aquáticos desta região nordestina, e em específico, no semiárido paraibano, que registrem sobre o conhecimento das comunidades ícticas e os processos ecológicos envolvidos (MALTCHIK, 1996; MALTCHIK; MEDEIROS, 2006; MARINHO et al., 2006; MEDEIROS et al., 2006; MONTENEGRO, 2007; CHAVES et al., 2009).

Diante disto, faz-se de grande importância a realização de estudos que visem o conhecimento da riqueza de espécies e dos aspectos da estrutura populacional e reprodução da ictiofauna de poças temporárias e semipermanentes do rio Taperoá, com o intuito de conhecer o seu papel na conservação da 
diversidade e produtividade pesqueira, para posterior intervenção em prol da qualidade da água bem como, para os diversos usos pela população.

\section{Material e Métodos}

O presente estudo foi realizado em poças do rio Taperoá no período de seca, dos anos de 2002 a 2004 (maio a dezembro de 2002 a 2004), na sub-bacia do rio Taperoá, região dos Cariris Velhos da Paraíba. Essa região é classificada como semiárida, desde que a precipitação anual não ultrapassa $800 \mathrm{~mm}$.
O rio Taperoá é um rio de regime temporário, cujo sedimento é predominantemente composto por areia, de solo raso e pouco permeável, que possui baixa capacidade de armazenamento de água (MEDEIROS, 1999), o que leva ao seu caráter intermitente e influencia na formação de algumas poças, durante o período de estiagem.

As coletas de peixes foram realizadas em cinco pontos ao longo do rio Taperoá, incluindo um poço artificial e quatro poças naturais próximas à cidade de São João do Cariri, PB (Figura 1). O poço - P1 (também chamado de poço São João - coordenadas $7^{\circ} 23$ '28'S

FIGURA 1: Localização da sub-bacia do rio Taperoá e área de amostragem das poças temporárias no rio Taperoá, região semiárida da Paraíba.

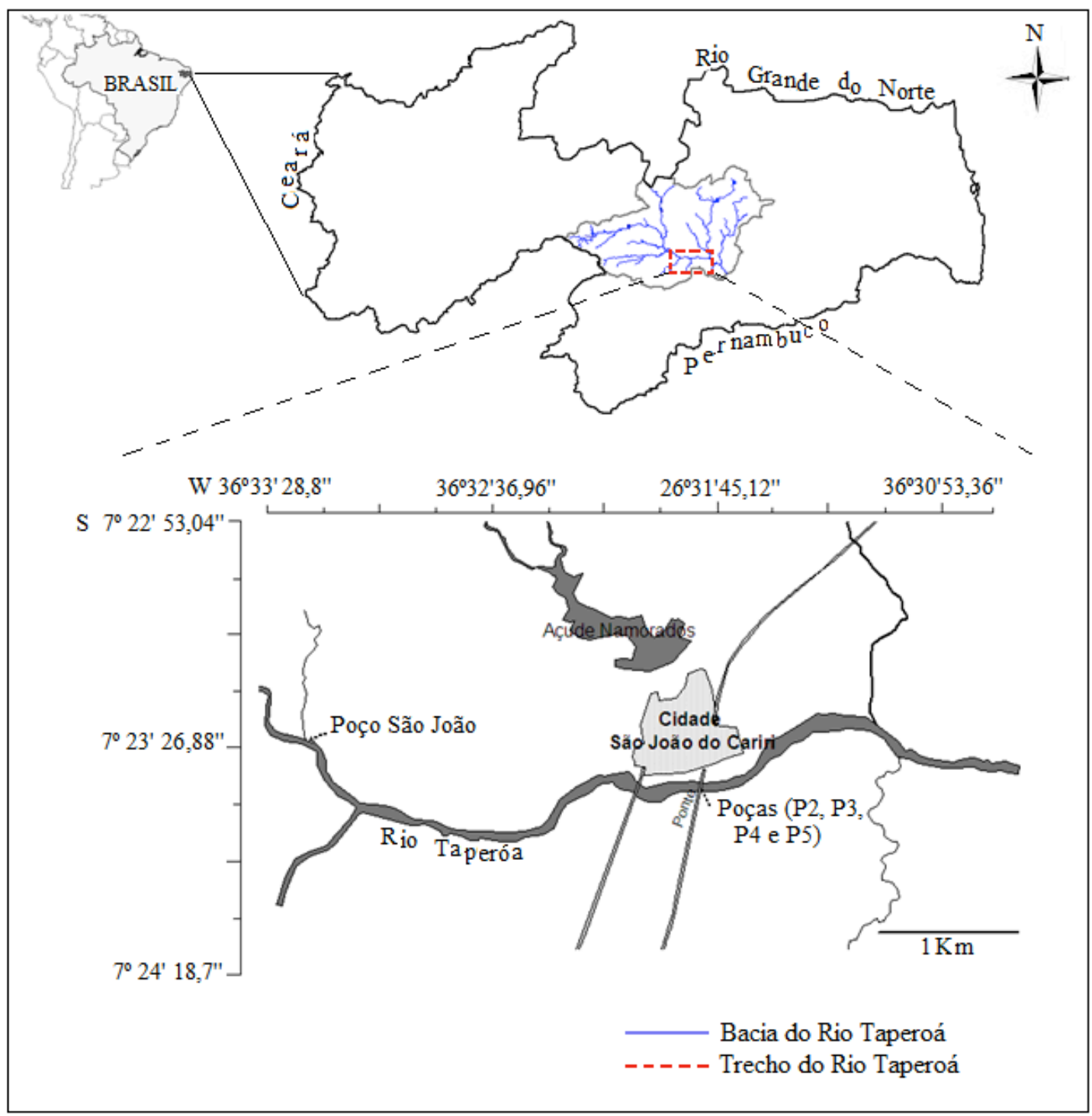


e $\left.36^{\circ} 33^{\prime} 20^{\prime \prime} \mathrm{W}\right)$ é um ambiente artesanal que recebe influência do rio Taperoá, pois localiza-se junto ao curso deste, com tamanho aproximado de $50 \mathrm{~m}^{2}$. As poças 2, 3, 4 e 5 (P2, P3, P4 e P5) foram localizadas logo abaixo da ponte do rio Taperoá em São João do Cariri (coordenadas 7²3'38”S e 36³1'46” W), possuindo extensão máxima de $10 \mathrm{~m}^{2}$. A poça 2 é uma poça temporária que permaneceu durante todo o período de estiagem. As poças temporárias 3, 4 e 5, secaram rapidamente, durando apenas um ou dois meses, e por isso não foram incluídas nas análises de variação temporal (coeficiente de variação) da abundância e riqueza de espécies.

Foram utilizadas tarrafas (malhas de 15 e $30 \mathrm{~mm}$ ) e puçás para a coleta dos peixes. As coletas foram realizadas no período diurno e o esforço de pesca foi padronizado para a coleta de dados. Após a coleta, os exemplares foram acondicionados em gelo. Em laboratório, foi realizada a triagem e biometria dos exemplares, para a determinação da estrutura populacional das espécies. Em seguida, as espécies foram identificadas, segundo Britiski (1972), Nelson (1984) e Nakatani et al. (2001).

Para a descrição da estrutura da comunidade íctica, foi determinada a riqueza de espécies (como número total de espécies encontradas), sendo aplicados os índices de diversidade de Shannon e Equitabilidade de Pielou. Para o cálculo dos índices de diversidade e equitabilidade, foi utilizado o software Biostat 5.0.

Para a determinação da atividade reprodutiva, foi realizada uma incisão abdominal nos exemplares, para observar as gônadas macroscopicamente, com o objetivo de determinar os estádios de maturação dos indivíduos, adotando-se a escala de maturidade (segundo VAZZOLER, 1996). Ao classificar os indivíduos em fase reprodutiva, foram considerados somente aqueles que apresentaram as gônadas maduras ou em maturação, ou que já haviam desovado (estádio esgotado).

Para investigar o grau de similaridade e dissimilaridade entre as poças, foi aplicada uma Análise de Cluster, por meio da distância euclidiana relativa, como medida de dissimilaridade (para a presença e ausência de espécies), utilizando-se o software Statistica 6.0.

Foi utilizado o coeficiente de variação (desvio padrão dividido pela média) para determinar a variabilidade na abundância das espécies ícticas ao longo do tempo, para as poças 1 e 2 . Foi escolhido o coeficiente de variação (CV), por ser bastante utilizado como estimativa de estabilidade em estudos biológicos (COTTINGHAN et al., 2001; PERSSON et al., 2001).

\section{Resultados}

\section{Níveis de precipitação entre os anos de 2002 a 2004}

No município de São João do Cariri/PB, a precipitação anual mostrou uma ampla variação, entre os anos de 2002 a 2004. O período de 2004 apresentou os maiores níveis de precipitação $(744,8 \mathrm{~mm})$, e os grandes açudes permaneceram cheios mesmo durante o período de estiagem. Durante o ano de 2003, o período chuvoso foi curto e pouco intenso, resultando num total anual de $323,5 \mathrm{~mm}$, caracterizando-se por um período de seca intensa. Em comparação, o ano de 2002 apresentou um total anual de precipitação de $641,6 \mathrm{~mm}$, sendo intermediário entre os anos de 2003 e 2004 (Figura 2).

FIGURA 2: Precipitação total durante o período de janeiro a dezembro nos anos de 2002 a 2004, no município de São João do Cariri/PB.

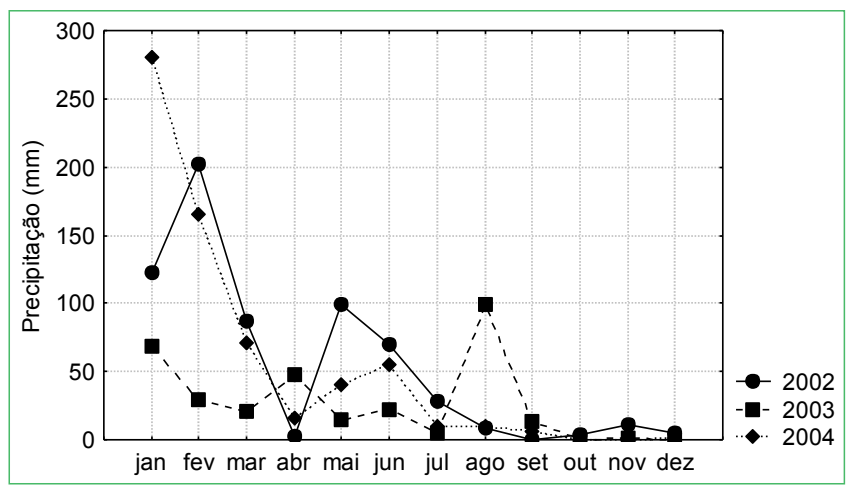

\section{Composição da ictiofauna registrada} nas poças

Foi coletado um total de 283 indivíduos, distribuídos em 13 espécies, nos cinco ambientes estudados (Tabela 1): Hoplias malabaricus (traíra); Steindachnerina notonota (saguiru); Astyanax bimaculatus (piaba); Oreochromis niloticus (tilápia - espécie exótica); Crenicichla menezesi (peixe-sabão); Prochilodus 
brevis (curimatã); Leporinus piau (piau); Hypostomus sp. (cascudinho); Cichlassoma orientale (cará); Psectrogaster rhomboides (branquinha); Poecilia vivipara (barrigudinho); Triportheus signatus (sardinha); e Characidium bimaculatus.
No poço São João, a composição foi representada por sete espécies, com $C$. orientale sendo a mais abundante $(33,3 \%)$, seguida por $H$. malabaricus $(27,4 \%)$ (Figura 3). O P2 foi a poça mais diversa e

TABELA 1: Lista sistemática de espécies de peixes identificadas nas poças do rio Taperoá, município de São João do Cariri/PB.

\begin{tabular}{|c|c|c|}
\hline Ordem & Família & Espécie \\
\hline Perciformes & Cichlidae & $\begin{array}{l}\text { Oreochromis niloticus (Linnaeus, 1758) } \\
\text { Cichlassoma orientale (Swainson, 1839) } \\
\text { Crenicichla menezesi Ploeg, } 1991\end{array}$ \\
\hline Siluriformes & Loricaridae & Hypostomus sp. Lacépède, 1803 \\
\hline Cyprinodontiformes & Poeciliidae & Poecilia vivipara $($ Bloch \& Schneider, 1801) \\
\hline \multirow[t]{7}{*}{ Characiformes } & Erythrinidae & Hoplias malabaricus (Block, 1974) \\
\hline & Curimatidae & $\begin{array}{l}\text { Steindachnerina notonota (Miranda-Ribeiro 1937) } \\
\text { Psectrogaster rhomboides Eigenmann \& Eigenmann, } 1889\end{array}$ \\
\hline & Characidae & Astyanax bimaculatus (Linnaeus, 1758) \\
\hline & Crenuchidae & Characidium bimaculatum Fowler, 1941 \\
\hline & Prochilodontidae & Prochilodus brevis Steindachner, 1874 \\
\hline & Triportheinae & Triportheus signatus (Garman, 1890) \\
\hline & Anostomidae & Leporinus piau Fowler, 1941 \\
\hline
\end{tabular}

FIGURA 3: Abundância relativa das espécies de peixes encontradas nas poças do rio Taperoá, município de São João do Cariri, PB: a) poço São João; b) poça 2 ; c) poça 3 ; d) poça 4 ; e) poça 5 .

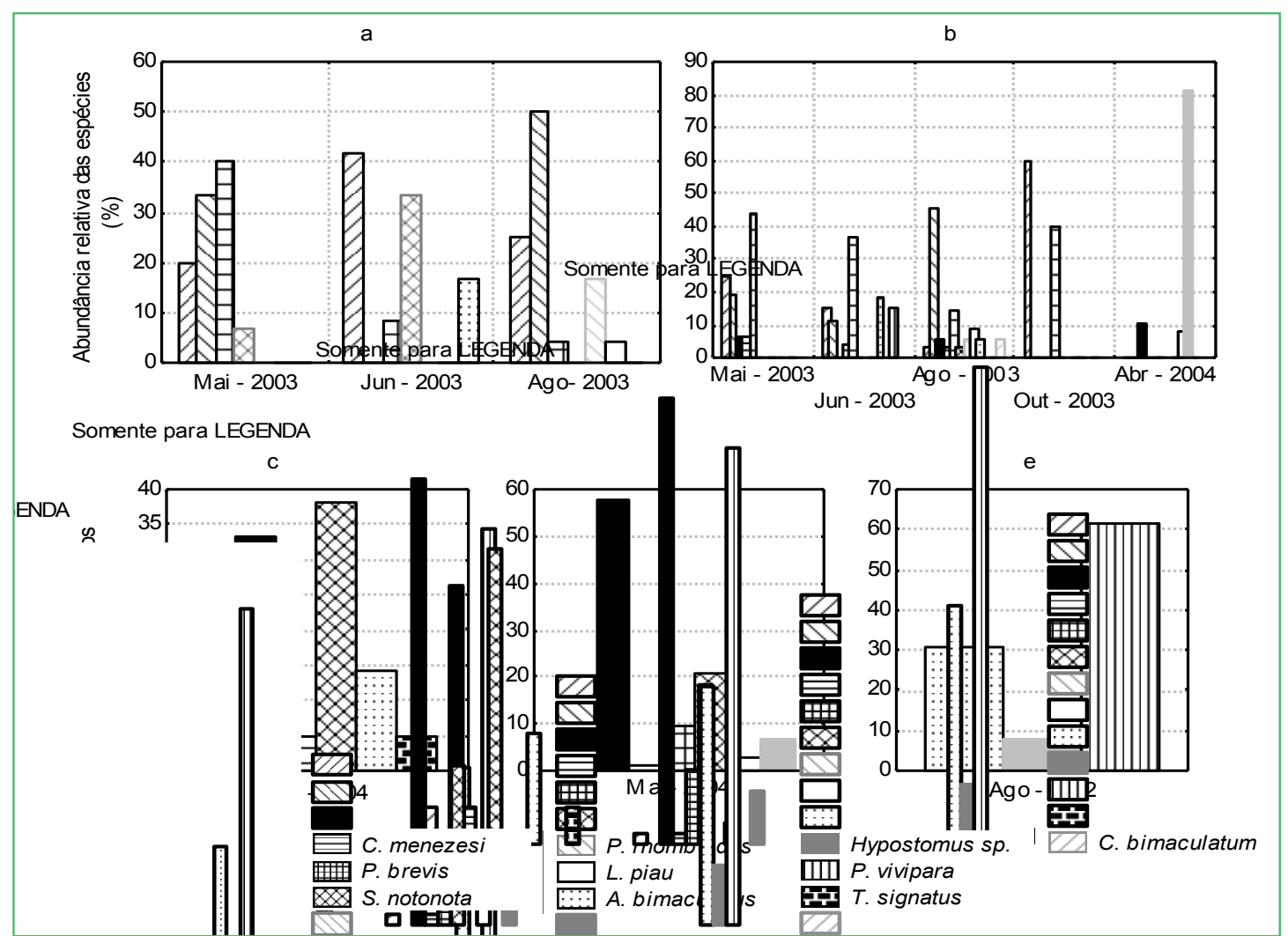


apresentou um total de 12 espécies, com as espécies mais abundantes: Hypostomus sp. (29,7\%); P. brevis (18,3\%); e C. orientale (16,7\%) (Tabela 2; Figura 3a). Ao final do período de estiagem de 2003, P2 apresentou baixos índices de diversidade (Tabela 2), pois apenas duas espécies foram encontradas (H. malabaricus e $P$. brevis), enquanto no ano de 2004, apenas três espécies foram registradas: $O$. Niloticus; Hypostomus sp.; e $A$. bimaculatus (Figura 3b).

A poça 3 foi representada por seis espécies, sendo a mais abundante $S$. notonota $(38,9 \%)$, seguida pela espécie exótica $O$. niloticus $(33,3 \%)$ (Figura 3c). Na poça 4, foram coletadas sete espécies, com a espécie introduzida O. niloticus contando com $57,5 \%$ do total dos indivíduos coletados, seguida por S. notonota (20,5\%) (Figura 3d). Em comparação, foram encontradas apenas três espécies na poça 5: P. vivipara (61,5\%); A. bimaculatus $(30,7 \%)$; e Hypostomus sp. (7,6\%) (Figura 3e).

TABELA 2: Riqueza (S), diversidade de Shannon-Wiener $\left(\mathrm{H}^{\prime}\right)$ e homogeneidade (J') das espécies encontradas nas poças do rio Taperoá.

\begin{tabular}{llcccc}
\hline & S & H' & J' & $\begin{array}{c}\text { Espécie } \\
\text { dominante }\end{array}$ \\
\hline P 1 & Maio/2003 & 4 & 1,23 & 0,89 & P. brevis \\
& Junho/2003 & 4 & 1,23 & 0,89 & H. malabaricus \\
& Agosto/2003 & 5 & 1,25 & 0,78 & C. orientale \\
P 2 & Maio/2003 & 5 & 1,36 & 0,85 & P. brevis \\
& Junho/2003 & 6 & 1,61 & 0,89 & P. brevis \\
& Agosto/2003 & 10 & 1,8 & 0,78 & C. orientale \\
& Outubro/2003 & 2 & 0,29 & 0,97 & H. malabaricus \\
& Abril/2004 & 3 & 0,83 & 0,75 & Hypostomus sp. \\
P 3 & Maio/2004 & 6 & 1,44 & 0,80 & S. notonota \\
P 4 Maio/2004 & 7 & 1,26 & 0,65 & O. niloticus \\
P 5 & Agosto/2002 & 3 & 0,85 & 0,78 & A. bimaculatus \\
\hline
\end{tabular}

A abundância de indivíduos de cada espécie apresentou alta variabilidade entre os meses de coleta nos pontos 1 e 2 (Tabela 3); porém, o coeficiente de variação da riqueza e da abundância total dos indivíduos foi baixo entre os meses de coleta no $\mathrm{P} 1(\mathrm{CV}$ riqueza $=0,133 ; \mathrm{CV}$ abundância $=0,367)$, e moderadamente flutuante no P2 $(\mathrm{CV}$ riqueza $=0,589 ; \mathrm{CV}$ abundância $=0,634)$.
TABELA 3: Valores da abundância média dos indivíduos nos pontos 1 e 2 e coeficiente de variação $(\mathrm{CV})$ da abundância de cada espécie, da abundância total e riqueza específica entre os meses de coleta.

\begin{tabular}{lcccc}
\hline \multicolumn{1}{c}{ Espécies } & P1 & P2 & CV P1 & CV P2 \\
\hline H. malabaricus & 27,45 & 9,16 & 0,392 & 1,178 \\
O. niloticus & - & 6,1 & - & 0,999 \\
C. menezesi & - & 2,29 & - & 1,034 \\
C. orientale & 33,33 & 16,7 & 0,916 & 1,247 \\
Hypostomus sp. & - & 29,7 & - & 2,236 \\
P. vivipara & - & 3,05 & - & 2,236 \\
S. notonota & 9,8 & 0,76 & 1,322 & 2,236 \\
P. rhomboides & 7,84 & 1,52 & 1,732 & 2,236 \\
A. bimaculatus & 3,92 & 8,39 & 1,732 & 1,171 \\
C. bimaculatum & - & 1,52 & - & 2,236 \\
P. brevis & 15,68 & 18,32 & 1,119 & 0,702 \\
L. piau & 1,96 & 2,29 & 1,732 & 2,236 \\
\hline Abundância total & & & 0,367 & 0,634 \\
Riqueza & & & 0,133 & 0,598 \\
\hline
\end{tabular}

De acordo com o grau de dissimilaridade encontrada entre as poças, por meio da distância euclidiana para a presença e ausência de espécies, o poço São João (P1) e o P2, sendo ambientes semipermanentes, foram mais semelhantes quanto à composição íctica, do que as outras poças que secaram rapidamente (Figura 4).

FIGURA 4: Dendograma de Dissimilaridade Euclidiana entre as poças, para a presença e ausência de espécies.

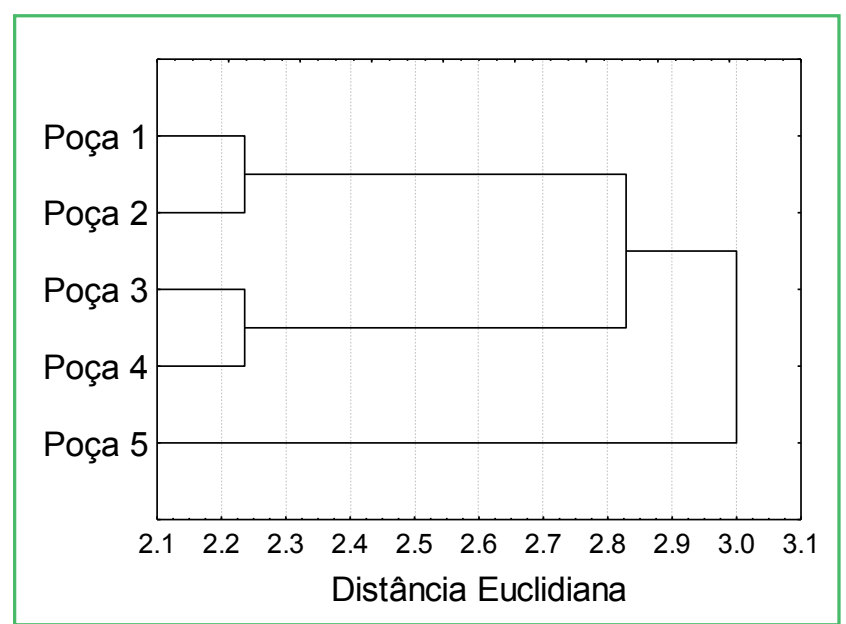




\section{Estrutura populacional das espécies}

Dentre as espécies encontradas, o comprimento padrão (CP) mínimo foi $1,0 \mathrm{~cm}$ (P. vivipara), e o CP máximo foi $22,4 \mathrm{~cm}$ ( $H$. malabaricus). Observa-se que o CP médio das espécies foi $7,25 \mathrm{~cm}$, e espécies como $H$. malabaricus, $O$. niloticus, $C$. menezesi, $S$. notonota, $P$. rhomboides, $P$. brevis e $L$. piau apresentaram CP médio acima de $7,25 \mathrm{~cm}$, com maior abundância das classes de comprimento acima de $6 \mathrm{~cm}$ (Figura 5; Tabela 4). A maioria das espécies (S. notonota, H. malabaricus, $L$. piau, C. orientale, P. brevis, e P. rhomboides) apresentou grande abundância de indivíduos em algum estágio de atividade reprodutiva (acima de 50\%) (Tabela 4). A espécie $O$. niloticus foi a que apresentou menor taxa de indivíduos em reprodução $(35,7 \%)$.

Não houve diferença significativa de tamanho das espécies de importância econômica entre as poças (P2, P3, P4 e 5) e o Poço São João (H. malabaricus: $\mathrm{t}$-value $=1,871, \mathrm{GL}=26, \mathrm{p}=0,075 ; P$. brevis: $\mathrm{t}-\mathrm{value}=$ $-1,919, \mathrm{GL}=35, \mathrm{p}=0,063 ;$ S. notonota: $\mathrm{t}-\mathrm{value}=-0,53$, $\mathrm{GL}=28, \mathrm{p}=0,595)$.
FIGURA 5: Classes de tamanho das principais espécies encontradas nas poças do rio Taperoá. HM - $H$. malabaricus; $\mathrm{ON}-$ O. niloticus; $\mathrm{PB}-$ P. brevis; $\mathrm{CO}-$ $C$. orientale; $\mathrm{SN}-S$. notonota; $\mathrm{PR}-P$. rhomboides; $\mathrm{AB}-A$. bimaculatus; $\mathrm{CM}-C$. menezesi; LP $-L$. piau; Hp - Hipostomus sp.; $\mathrm{PV}-\mathrm{P}$. vivipara.

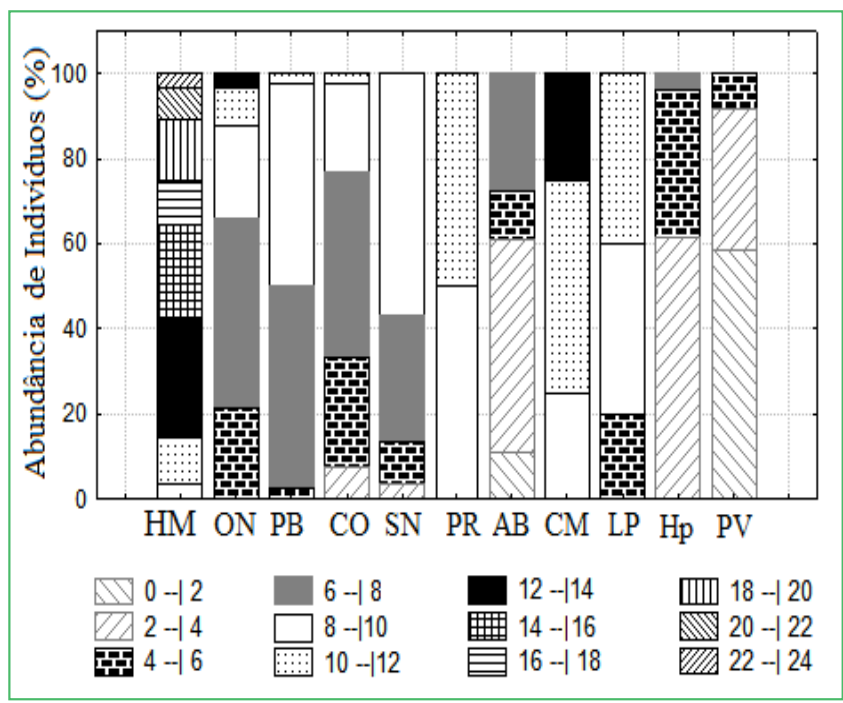

TABELA 4: Espécies de peixes capturadas, número de indivíduos $(\mathrm{N})$, comprimento padrão $(\mathrm{CP})$ médio $( \pm$ desvio padrão), mínimo e máximo, número de indivíduos com os estádios gonadais imaturos (Im,), em maturação (Emat.), maduros (Mad.) e esgotados (Esg.), porcentagem de indivíduos em estágio reprodutivo (\% IR - em maturação, maduro e esgotado) e menor tamanho de indivíduo em estágio reprodutivo ( $\mathrm{R}$ mín).

\begin{tabular}{lccccccccc}
\hline Espécies & $\mathbf{N}$ & CP Médio & CP mín - max. & Im. & Emat. & Mad. & Esg. & \% IR & R mín. \\
\hline H. malabaricus & 28 & $15,01(3,3)$ & $8,6-22,4$ & 5 & 13 & 2 & 2 & 77,2 & 8,6 \\
O. niloticus & 56 & $7,61(1,9)$ & $5,1-13,4$ & 27 & 7 & 5 & 3 & 35,7 & 8,0 \\
C. menezesi & 4 & $10,52(1,8)$ & $8,0-10,4$ & 1 & 0 & 0 & 0 & - & - \\
C. orientale & 39 & $6,82(1,6)$ & $3,1-10,7$ & 6 & 8 & 2 & 3 & 68,4 & 5,5 \\
Hypostomus sp. & 45 & $3,20(1,0)$ & $2,2-5,3$ & 10 & 0 & 0 & 0 & - & - \\
P. vivipara & 12 & $2,02(1,2)$ & $1,0-4,8$ & 0 & 0 & 1 & 0 & - & - \\
S. notonota & 30 & $8,04(1,4)$ & $3,7-9,6$ & 1 & 0 & 0 & 24 & 96,0 & 5,5 \\
P. rhomboides & 4 & $9,97(0,5)$ & $9,4-10,5$ & 1 & 1 & 0 & 0 & 50,0 & 10,5 \\
A. bimaculatus & 18 & $3,8(1,9)$ & $1,9-7,9$ & 9 & 2 & 3 & 2 & 43,7 & 4,6 \\
C. bimaculatum & 2 & $3,2(0,2)$ & $3,0-3,4$ & - & - & - & - & - & - \\
P. brevis & 38 & $8,02(1,1)$ & $5,7-10,8$ & 8 & 3 & 7 & 4 & 63,6 & 7,3 \\
T. signatus & 1 & 6,9 & - & - & - & - & - & - & - \\
L. piau & 6 & $9,2(3,3)$ & $4,4-13,6$ & 1 & 2 & 0 & 1 & 75,0 & 10,4 \\
\hline TOTAL & $\mathbf{2 8 3}$ & $\mathbf{7 , 2 5}$ & $\mathbf{1 , 0 - 2 2 , 4}$ & $\mathbf{6 9}$ & $\mathbf{3 6}$ & $\mathbf{2 0}$ & $\mathbf{3 9}$ & $\mathbf{5 7 , 9}$ & $\mathbf{4 , 6}$ \\
\hline
\end{tabular}




\section{Discussão}

As variações anuais e mensais na precipitação e no fluxo de água promovem grandes alterações nas variáveis físico-químicas, que podem influenciar diretamente às comunidades ícticas. Durante o período estudado, o ano de 2004 apresentou os maiores níveis de precipitação, que segundo Crispim et al. (2006), representou o maior período chuvoso dos últimos 40 anos, sendo precedido por um período de seca intensa em 2003.

Ocorrem cerca de 240 espécies de peixes na Caatinga brasileira (ROSA et al., 2008); porém, no presente estudo apenas 13 espécies foram capturadas nas poças do rio Taperoá. Isso foi similar ao número de espécies (15) que foi encontrado no rio Taperoá por Medeiros e Maltchik (2001a). Outros estudos também demonstraram a baixa riqueza de espécies de peixes nos ambientes da Caatinga (MEDEIROS; MALTCHIK, 2001b; MALTCHIK; MEDEIROS, 2006), corroborando a pesquisa de Paiva (1978), que realizou uma estimativa de apenas 15 a 20 espécies de peixes existentes em cada rio ou riacho das regiões semiáridas do Brasil. O baixo número de espécies nos ambientes semiáridos é um reflexo das limitações impostas pelo longo período de estiagem (MEDEIROS; MALTCHIK, 2001a).

A presença de espécies exóticas também pode ser um fator que influencia na redução da riqueza de espécies (VITOUSEK et al., 1997). Diversos são os estudos que indicam os efeitos de tilápias do gênero Oreochromis na redução da captura de espécies nativas (MENESCAL, 2002; CANONICO et al., 2005; ATTAYDE et al., 2011). Entretanto, no presente estudo, pouco pode ser concluído em relação à presença da espécie introduzida O. niloticus, desde que dados anteriores à introdução sobre a riqueza e diversidade de espécies nativas não existem. Além disso, vários estudos já tinham relatado sobre a baixa riqueza de espécies encontrada em diferentes ambientes aquáticos nas regiões semiáridas do Brasil (PAIVA, 1978; MEDEIROS; MALTICHIK, 2001b). Mesmo assim, uma alta abundância de tilápias foi encontrada nas poças P3 e P4, sendo possivelmente um reflexo da abundância desta espécie no rio Taperoá.

No presente estudo, todas as espécies apresentaram alta variabilidade em sua abundância entre os meses de coleta; porém, a riqueza de espécies demonstrou baixa flutuação no poço São João (P1) e moderada flutuação na poça 2. As diferenças na abundância e riqueza de espécies podem ser causadas pelo fluxo e variação na qualidade da água, desde que no P2, ao final do período de estiagem, foram encontradas apenas duas espécies de peixes. Medeiros e Maltichik (2001a; 2001b) e Maltichik e Medeiros (2006) encontraram a maior estabilidade na diversidade de espécies durante o período de seca. Ao mesmo tempo, esses estudos encontraram baixa variação da abundância de espécies entre os meses de coleta, que pode ser explicado pelas difíceis condições ambientais durante a seca, tornando-se algumas espécies dominantes, e, consequentemente, diminuindo a diversidade.

A variabilidade na dominância das espécies parece estar ligada as condições severas do período seco. No início da estiagem, a espécie P. brevis dominou a ictiofauna nos pontos 1 e 2. Com a intensificação da seca, espécies mais resistentes como H. malabaricus, o ciclídeo C. orientale e espécies do gênero Hypostomus dominaram a ictiofauna das poças. Semelhantemente, Medeiros e Maltchik (2001a; 2001b) também encontraram maior dominância de Hypostomus sp. e do ciclídeo introduzido $O$. niloticus na mesma região. Além disso, deve existir uma variação anual na riqueza de espécies em poças, relacionada à distribuição aleatória dos indivíduos durante a formação das poças, onde apenas alguns indivíduos acabam colonizando as poças, enquanto diversos outros indivíduos são levados pela correnteza do rio.

A estrutura populacional revelou que as espécies de importância econômica para a região semiárida (P. brevis, P. rhomboides, L. piau, O. niloticus, H. malabaricus e $S$. notonota) apresentaram tamanhos médios acima de $7 \mathrm{~cm}$, e mais de $50 \%$ dos indivíduos encontravam-se em atividade reprodutiva (com as gônadas em maturação, maduras ou esgotadas, com exceção da espécie $O$. niloticus), indicando a importância da formação das poças para a permanência das espécies. Diferentemente, Medeiros (1999), em seus estudos sobre o rio Taperoá, encontrou baixos valores para a atividade reprodutiva durante o período de estiagem, relacionando o período reprodutivo à presença de fluxo de água. 
Para Alkins-Koo (2000), a atividade reprodutiva parece ser desencadeada pelas inundações que ocorrem antes da formação das poças, e que estas podem ser importantes para o recrutamento das espécies. Entretanto, os resultados encontrados no presente estudo podem estar relacionados ao estresse de limitação de área aquática das poças, desencadeando a reprodução como instinto de sobrevivência. Segundo Paiva (1978), as condições hostis das poças, à medida que o período de estiagem se intensifica, afetam as comunidades de peixes e podem diminuir a idade de maturação. A exemplo disto, a traíra (H. malabaricus) apresentou indivíduos em reprodução com $8,6 \mathrm{~cm}$, enquanto em ambientes não perturbados foram encontrados indivíduos atingindo a primeira maturação gonadal a partir dos $21 \mathrm{~cm}$ (MARTINS, 2009). Além disso, o cará (C. orientale) e o curimatã (P. brevis) apresentaram indivíduos em reprodução a partir dos 5,5 e 7,3cm respectivamente, enquanto Souza (2010), para as mesmas espécies, encontrou comprimentos da primeira maturação gonadal de $7,7( \pm 0,97)$ e $18,6( \pm$ 0,07), respectivamente.

Embora o poço São João seja um ambiente artificial, a presença e ausência de espécies foi semelhante à poça 2 , uma poça natural que permaneceu alagada o ano todo. Além disso, o poço São João não apresentou diferença significativa de tamanho das espécies de importância econômica com as demais poças. Apesar disto, o Poço São João apresentou maior estabilidade da abundância de indivíduos e riqueza de espécies, quando comparado à poça 2. A maior estabilidade pode ser uma consequência do maior tamanho do poço e, assim, menor variação no fluxo de água. Medeiros e Maltchik (2001a) observaram que a formação de grandes poças durante o período de estiagem promoveram maior estabilidade. Assim, embora o Poço receba as águas do rio Taperoá, como as poças, o tamanho dos ambientes pode ser um fator importante para a manutenção das espécies.

Em diversos ambientes, como as planícies de inundação do Pantanal e da Amazônia, as formações de poças e lagoas marginais são reconhecidas como importantes locais de abrigo e berçário para espécies de peixes, sustentando a diversidade e produção pesqueira (PETRERE, 1983; MUNIZ, 2005).
Em regiões semiáridas, como a Caatinga nordestina, poucos estudos tem sido realizados sobre a importância das poças para manutenção das espécies de peixes. Apesar disto, Luz et al. (2009) observaram a importância de lagoas marginais ao rio São Francisco como abrigo e descanso para a ictiofauna e sustento pesqueiro.

Segundo Medeiros (1999), o período de seca representa uma fase de crescimento de jovens e as poças constituem áreas de refúgio para a manutenção das espécies. Embora o tamanho de cada poça seja geralmente pequeno, com a capacidade de suportar poucos indivíduos até o final da estiagem, as poças permanentes e temporárias são encontradas em grande quantidade ao longo dos rios na região semiárida do Brasil.

O presente estudo indica a importância das poças para a conservação dos estoques de peixes, desde que fornece abrigo e alimento para diversas espécies. Além disso, os resultados demonstram que as condições severas aparentemente estimulam a reprodução, como forma de sobrevivência, garantindo o recrutamento das espécies e a produção pesqueira.

\section{Agradecimentos}

Agradecemos ao $\mathrm{CNPq}$ pelo suporte financeiro através do programa Ecológico de Longa Duração (PELD-Caatinga: estrutura e funcionamento).

\section{Referências}

ABÍLIO, F. J. P. Gastrópodes e outros invertebrados bentônicos do sedimento litorâneo e associado a macrófitas aquáticas em açudes do semiárido paraibano, nordeste do Brasil. 2002. 179 f. Tese (Doutorado em Ecologia e Recursos Naturais) - Universidade Federal de São Carlos, São Carlos. 2002.

ABÍLIO, F. J. P.; RUFFO, T. L. M.; SOUZA, A. H. F. F.; FLORENTINO, H. S.; OLIVEIRA, E. T. J.; MEIRELES, B. N.; SANTANA, A. C. D. Macroinvertebrados bentônicos como bioindicadores de qualidade ambiental de corpos aquáticos da caatinga. Oecologia Brasiliensis, Rio de Janeiro, v. 11, n. 3, p. 397-409, 2007.

ALKINS-KOO, M. Reproductive timing of fishes in a tropical intermittent stream. Environmental Biology of Fishes, Dordrecht, v. 57, p. 49-66, 2000.

ATTAYDE, J. L.; BRASIL, J.; MENESCAL, R. A. Impacts of introducing Nile tilapia on the fisheries of a trop ical reservoir in 
North-east ern Brazil. Fisheries Management and Ecology, East Yorkshire, v. 18, p. 437-443.

BARBOSA, C. B. Estabilidade de comunidades ribeirinhas no semiárido brasileiro. 1998. 124 f. Dissertação (Mestrado em Desenvolvimento e Meio Ambiente) - Universidade Federal da Paraíba, João Pessoa. 1998.

BRITISKI, H. A. Peixes de água doce do Estado de São Paulo. In: BRANCO, M. S. (Ed.). Poluição e Piscicultura. São Paulo: Faculdade de Saúde Pública da USP, 1972. p. 79-108.

CANONICO, G. C.; ARTHINGTON, A.; McCRARY, J. K.; THIEME, M. L. The effects of introduced tilapias on native biodiversity. Aquatic Conservation: Marine and Freshwater Ecosystems, Malden, v. 15, p. 463-483, 2005.

CHAVES, M. F.; TORELLI, J. E. R. S.; TARGINO, C. H.; CRISPIM, M. C. Dinâmica reprodutiva e estrutura populacional de Hoplias aff. malabaricus (Bloch, 1794) (Characiformes, Erythrinidae), em açude da Bacia do Rio Taperoá, Paraíba. Biotemas, Florianópolis, v. 22, n. 2 , p. $85-89,2009$.

COTTINGHAM, K. L.; BROWN, B. L.; LENNON, J. T. Biodiversity may regulated the temporal variability of ecological systems. Ecology Letters, Montpellier, v. 4, p. 72-85, 2001.

CRISPIM, M. C.; RIBEIRO, L. L.; GOMES, S. E. M.; FREITAS, G. T. P.; SERPE, F.R. Comparision of different kind of semiarid aquatic environments based on zooplankton communities. Revista de Biologia e Ciências da Terra, Campina Grande, v. 6, n. 1 (suplemento especial), p. 98-111, 2006.

LACERDA, A. V. A semiaridez e a gestão em bacias hidrográficas: visões e trilhas de um divisor de idéias. João Pessoa: EDUFPB, 2003. 164 p.

LUZ, S. C. S.; EL-DEIR, A. C. A.; FRANÇA, E. J.; SEVERI, W. Estrutura da assembléia de peixes de uma lagoa marginal desconectada do rio, no submédio Rio São Francisco, Pernambuco. Biota Neotropica, Campinas, v. 9, n. 3, p. 117-129, 2009.

MALTCHIK, L. Nossos rios temporários, desconhecidos mas essenciais. Ciência Hoje, Rio de Janeiro, v. 21, p. 63-65, 1996.

MALTCHIK, L. Ecologia de rios intermitentes tropicais. In: POMPÊO, M. L. M. (Ed.). Perspectivas da Limnologia no Brasil. São Luis: Gráfica e Editora União, 1999. p. 77-89.

MALTCHIK, L.; MEDEIROS, E. S. F. Diversidade, estabilidade e atividade reprodutiva de peixes em uma poça fluvial permanente no leito de um riacho efêmero, riacho Avelós, Paraíba, Brasil. Revista de Biologia e Ciências da Terra, Campina Grande, v. 6, n. 1(suplemento especial), p. 20-28, 2006.

MARINHO, R. S. A.; TORELLI, J. E. R. S.; SILVA, A. S.; RIBEIRO, L. L. Biodiversidade de peixes do semiárido paraibano. Revista de Biologia e Ciências da Terra, Campina Grande, v. 6, n. 1 (suplemento especial), p. 112-121, 2006.

MARTINS, J. M. E. Biologia de Hoplias malabaricus (Bloch, 1794) (Characiformes, Erythrinidae) na represa de Capim Branco I, Rio Araguari, MG. 2009. 84 f. Dissertação (Mestrado em Ecologia e Conservação dos Recursos Naturais) - Universidade Federal de Uberlândia, Uberlândia. 2009.

MATTHEWS, W. J. Patterns in freshwater fish ecology. Massachussets: Chapman \& Hall, 1998. 784 p.

MEDEIROS, E. S. F. Efeito das perturbações hidrológicas na diversidade, estabilidade e atividade reprodutiva de peixes em rios intermitentes do semiárido brasileiro. 1999. $98 \mathrm{f}$. Dissertação (Mestrado em Ciências Biológicas) - Universidade Federal da Paraíba, João Pessoa. 1999.

MEDEIROS, E. S. F.; MALTCHIK, L. Influence of hydrological disturbance on reproduction of a fish community in an intermittent stream from Brazilian semiarid region. Verhandlungen der Internationalen Vereinigung für theoretische und Angewandte Limnologie, Stuttgart, v. 27, p. 906-911, 2000.

MEDEIROS, E. S.; MALTCHIK, L. Diversity and stability of fishes (TELEOSTEI) in a temporary river of the Brazilian semiarid region. Iheringia Série Zoológica, Porto Alegre, v. 90, p. 157-166, 2001a.

MEDEIROS, E. S. F.; MALTCHIK, L. Fish assemblage stability in an intermittently flowing stream from the Brazilian semiarid region. Austral Ecology, Alice Springs, v. 26, p. 156-164, 2001 b.

MEDEIROS, E. S. F.; RAMOS, R. T. C.; RAMOS, T. P. A.; SILVA, M. J. Spacial variation in reservoir fish assemblages along semiarid intermittent river, Curimataú River, northeastern Brazil. Revista de Biologia e Ciências da Terra, Campina Grande, v. 6, n. 1 (suplemento especial), p. 29-39, 2006.

MENESCAL, R. A. Efeitos da introdução da tilápia do Nilo, Oreochromis niloticus (Linneaus, 1978), sobre o desembarque pesqueiro no açude Marechal Dutra, Acari, RN. 2002. 50 f. Dissertação (Mestrado em Bioecologia Aquática) - Universidade Federal do Rio Grande do Norte, Natal. 2002.

MONTENEGRO, A. K. A. Bioecologia da ictiofauna do açude Taperoá II, semiárido Paraibano, Brasil. 2007. 162 f. Dissertação (Mestrado em Ciências Biológicas) - Universidade Federal da Paraíba, João Pessoa. 2007.

MUNIZ, C. C. Composição da comunidade íctica em área limnética, relacionada ao ciclo hidrológico nas baías da Salobra e Negra, no Pantanal de Cáceres - MT. 2005. 81 f. Dissertação (Mestrado em Ecologia e Conservação da Biodiversidade)Universidade Federal de Mato Grosso, Cuiabá. 2005.

NAKATANI, K.; AGOSTINHO, A. A.; BAUMGARTNER, G.; BIALETZKI, A.; SANCHES, P. V.; MAKRAKIS, M. C.; PAVANELLI, C. S. Ovos e larvas de peixes de água doce: desenvolvimento e manual de identificação. Maringá: EDUEM, $2001.378 \mathrm{p}$.

NELSON, J. S. Fishes of the world. New York: John Wiley and Sons Ldt, 1984. 523 p.

PAIVA, M. P. A ictiofauna e as grandes represas brasileiras. Revista DAE, São Paulo, v. 38, n. 116, p. 49-56, 1978.

PERSSON, A.; HANSSON, L. A.; BRÖNMARK, C.; LUNDBERG, P.; PETTERSSON, L. B.; GREENBERG, L.; NILSSON, P. A.; NYSTRÖM, P.; ROMARE, P.; TRANVIK, L. J. Effects of enrichment on simple aquatic food webs. American Naturalist, Chicago, v. 157, p. 654-669, 2001.

PETRERE, M. Jr. Relationships among catches, fishing effort and river morphology for eight rivers in Amazonas State (Brazil), during 1976-1978. Amazoniana, Plön, v. 8, n. 2, p. 281-296, 1983.

ROSA, R. S.; MENEZES, N. A.; BRITISKI, H. A.; COSTA, W. J. E.; GROTH, F. Diversidade, padrões de distribuição e conservação dos peixes da caatinga. In: LEAL, I. (Ed.). Ecologia e conservação da caatinga. Recife: EDUFPE, 2008. p. 135-180.

SOUZA, L. L. G. Ictiofauna do semiárido potiguar, Nordeste do Brasil: Composição, riqueza e ecologia reprodutiva de espécies 
endêmicas. 2010. 108 f. Tese (Doutorado em Ecologia e Recursos Naturais) - Universidade Federal de São Carlos, São Carlos. 2010.

VAZZOLER, A. E. A. D. M. Biologia da reprodução de peixes teleósteos: teoria e prática. Maringá: EDUEM, 1996. 169 p.

VIEIRA, A. C. B.; RIBEIRO, L. L.; SANTOS, D. P. N.; CRISPIM, M. C. Correlation between the zooplanktonic community and environmental variables in a reservoir from the Northeastern semiarid. Acta Limnologica Brasiliensia, Botucatu, v. 21, n. 3, p. 349-358, 2009.

VITOUSEK, P. M.; MOONEY, H. A.; LUBCHENCO, J.; MELILLI, J. M. Human domination of earth's ecosystems. Science, New York, v. 227, p. 494-499, 1997. 